\title{
Survey the role of in vivo and in vitro condition to expiration of nano structure para crystalline layer gene in bacteria
}

\author{
Shila Jalalpoor
}

\section{Lecture of Microbiology, Shahreza Branch, Islamic Azad University, and Membership of young researchers club, Iran} shilla.jalalpoor@yahoo.com, jalalpour@iaush.ac.ir

\begin{abstract}
Para crystalline layer is a monomolecular outermost protein layer in bacteria and archiae, credit of protein or glycoprotein subunits and has crystalline biopolymer structure. Para crystalline -layer protects bacteria to phagocytosis and prohibit the entry of some biomolecule -for example antibiotics- and adhesion to matrix proteins, is one of virulence agents and layer producer. Para crystalline layer have application potential in biotechnology, molecular nanotechnology, and biomimetics. The research was performed with laboratory method in 2005-2007 years, in Azzahra hospital and Isfahan University. In this research, 26 Bacillus cereus strains were studied. Identification of bacteria, was performed with microbiological methods: staining, chemical test, using differential and selective media. Isolation of Bacillus cereus strains was performed on Selective Agar, and cultured in TSA, for 16 hours, under aerobic condition. Then subjected to the separation of surface proteins and electrophoresed along with molecular weight marker. Para crystalline layer in $B$. cereus has $97 \mathrm{KD}$ MW. Out of $26 B$. cereus strain, 14 strain produced Para crystalline layer and 12 strain don't have Para crystalline -layer. Out of 13 isolates from staff hand, 11 sample $(84 / 6 \%)$ and from 13 isolates from hospital surfaces, 1 sample $(7 / 7 \%)$ have produced Para crystalline layer.
\end{abstract}

Keywords: Para Crystalline Layer, Bacillus cereus, nosocomial infections

Introduction

Nosocomial infections (NIs) remain a major global concern. Overall, national prevalence rates have been described as ranging between 3.5 and $9.9 \%$. They lead to additional days of treatment, increase the risk of death, and increase treatment costs. Staff hands and hospital surfaces have important role in NIs (Kamp \& Kramer, 2004). The health-care environment contains a diverse population of microorganisms (Sehulster \& Raymond, 2003).

Microorganisms are present in great numbers in moist, organic environments, but some also can persist under dry conditions. Environmental source or means of transmission of infectious agents, the presence of the pathogen does not establish its causal role; its transmission from source to host could be through indirect means, e.g., via hand transfer. The surface would be considered one of the potential reservoirs for the pathogens. The most important and frequent mode of transmission of nosocomial infections, is divided into two subgroups: direct-contact transmission and indirectcontact transmission (Sehulster \& Raymond, 2003).

Direct-contact transmission involves a direct body surface-to-body surface contact and physical transfer of microorganisms between a susceptible host and an infected or colonized person. Direct-contact transmission also can occur between two patients, with one serving as the source of the infectious microorganisms and the other as a susceptible host. Indirect-contact transmission involves contact of a susceptible host with a contaminated intermediate object, usually inanimate, such as contaminated instruments, needles, or dressings, or contaminated gloves that are not changed between patients and staff hands.
Bacillus cereus bacteria are large spore forming, Gram-positive rod-shaped, facultative anaerobes. $B$. cereus strains are common in the environment and can be found in soil, dust, air, water, and on decaying. It has been regarded as a relatively nonpathogenic opportunist commonly associated with enterotoxin mediated diarrheal food poisoning. This organism has been increasingly isolated from serious nongastrointestinal infections including endocarditis, wound infection, osteomyelitis, oral cavity associated with infected root canals, periodontal pockets, bovine mastitis, severe systemic, pyogenic infections, gangrene, septic meningitis, cellulitis, panophyhalmitis, lung abscesses, infant death, and endocarditis and now $B$. cereus regarded one of nosocomial infections bacteria (Van der Zwet et al., 2000; Hilliard et al., 2003; Washington et al., 2006).

Survival spore forming bacteria on hands and surfaces in vegetative cells of can survive for at least $24 \mathrm{~h}$ on inanimate surfaces, and spores survive for up to 5 months. Surface structures are an important structural component of prokaryotic organisms and essential for many aspects of their life (Jalalpoor et al., 2007). B. cereus produces several potential virulence factors in addition to the toxins associated with gastrointestinal infections, and these factors are thought to playa role in non-gastrointestinal infections. These virulence factors include three hemolysins, three phospholipases, three different beta lactamases, extracellular collagenases, membrane-bound proteases, and para crystalline layer (Jalalpoor et al., 2007, Washington et al., 2006).

Nosocomial outbreaks of Bacillus infections have involved common-source spread from contaminated reservoirs in the environment. These sources have included contaminated hemodialyzers, bronchoscopes, 
Ommaya reservoirs, manual ventilation balloons, multiple-unit injectables, and contaminated diapers, gloves, and surgical bandages (Jalalpoor et al., 2007; Van der Zwet et al., 2000; Washington et al., 2006). All of the various surface components of a bacterial cell are important in its ecology since they mediate the contact of the bacterium with its environment, the only senses that a bacterium possesses result from its immediate contact with its environment.

It must use its surface components to assess the environment and respond in a way that supports its own existence and survival in that environment. In medical situations, the surface components of bacterial cells are major determinants of virulence for many pathogens. The surface properties of a bacterium are determined by the exact molecular composition of its membrane and cell envelope, including capsules, glycocalyx, para crystalline layer, peptidoglycan, LPS, and the other surface structures, such as flagella and pili or fimbriae. Over the past 3 decades of research, it has become apparent that one of the most common surface structures on bacteria are monomolecular Para crystalline arrays of proteinaceous subunits termed surface layer or para crystalline layer. Para crystalline layer is attached to the outermost portion of their cell wall. It consists of a single molecular layer composed of identical proteins or glycoproteins and in electron micrographs, has a pattern resembling floor tiles (Sara \& Uwe, 2000; Mesnage et al., 2001; Messner et al., 2008; Sara, 2001).

The para crystalline layer lattices can have oblique $(p 1, p 2)$ square (p4), or hexagonal (p3, p6) symmetry. Depending on the lattice type, one morphological unit consists of one, two, four, three, or six identical (glyco) protein subunits, respectively, and they exhibit center-tocenter spacings of approximately 2.5 to $35 \mathrm{~nm}$. Most para crystalline layers are 5 to $25 \mathrm{~nm}$ thick. It is now evident that para crystalline layers are the most common cell surface components of pathogen bacteria such as Lactobacillus sp., Rickettsia sp., Serratia sp., Caulobacter sp., Campylobactersp., Corynebacterium sp., Clostridium sp. and Bacillus sp. (Sara \& Uwe, 2000; Mesnage et al., 2001; Messner et al., 2008; Sara, 2001).

Because para crystalline layer lattices possess pores identical in size and morphology in the 2 to $8 \mathrm{~nm}$ range, occupying up to $70 \%$ of the surface area they work as precise molecular sieves, providing sharp cutoff levels for the bacterial cells. para crystalline layers from various Bacillaceae were shown to be suitable for the production of isoporous ultrafiltration membranes with well-defined molecular weight cutoffs. The para crystalline layer lattice and the pore areas of para crystalline layers contain functional groups (carboxylic acid, amine, and hydroxyl groups) which are aligned in well-defined positions and orientations.

The repetitive features of para crystalline layers have led to their use as immobilization matrices for binding of monolayers of functional molecules e.g., enzymes,
Vol. 5 No. S3 (Mar 2012)

ISSN: 0974- 6846 antibodies, antibiotics and immunogens in a geometrically well-defined way. This application potential has been exploited for the production of bioanalytical sensors, immunoassays, affinity microparticles, and affinity membranes. The para crystalline layer has been associated with a number of possible functions, these include the following:

1. The para crystalline layer protects bacteria from harmful enzymes (para crystalline layer from Bacillaceae were found to function as adhesion sites for cell-associated exoenzymes) and antimicrobial agents.

2. The para crystalline layer protects bacteria from changes in $\mathrm{pH}$.

3. The para crystalline layer protects bacteria from attack by bacterial parasites such as Bdellovibrio bacteriovorus, and from bacteriophages.

4. The para crystalline layer can function as an adhesin, enabling the bacterium to adhere to host cells and environmental surfaces, colonize, and resist flushing.

5. The para crystalline layer may contribute to virulence by protecting the bacterium against complement attack and phagocytosis.

6. The para crystalline layer may act as a as a coarse molecular sieve. para crystalline layers can contribute to virulence when they are present as a structural component of the cell envelope of pathogens (Sara \& Uwe, 2000; Mesnage et al., 2001; Messner et al., 2008; Sara, 2001).

Spread of para crystalline layer producer $B$. cereus strains in staff hand and hospital surfaces leads to the increase of antibiotic resistant NIs. The aims of this search was to survey frequency of para crystalline layers of Bacillus cereus strains in hospital surfaces and staff hands.

Materials and methods

\section{Sampling}

A total of 274 bacteria, 194 bacteria from hospital surfaces and 80 bacteria from staff hand were isolated of Azzahra-hospital during of 2005-2007 years. Hospital surfaces samples were randomly collected from high and low hospital contact surfaces with swab (Effective sampling of surfaces requires moistened swabs) in Tryptone Soya Agar (Merck) and staff hand samples, were randomly collected from staff hand in Blood Agar (Merck) via Fingerprint Technique (Jalalpoor et al., 2009; Sehulster \& Raymond, 2003).

\section{Bacterial strains}

Specimen grown on sheep blood and chocolate agars were incubated at $37^{\circ} \mathrm{C}$ under aerobic conditions. Bacillus as Gram-positive bacilli, the intracellular and cellfree spores do not stain by the Gram technique but can be visualized with the malachite green stain by which the spores will appear green. On SBA, colonies of $B$. cereus usually large, with a matte or granular texture, and most strains are beta hemolytic. The strains were identified based on colony morphology, Gram stain reaction, spore 
formation, and biochemical tests with the BioMerieux database system (Kotiranta et al., 1998; Kotiranta et al.,1999).

\section{Detection of Para Crystalline Layer}

For the examination of surface proteins, $16 \mathrm{~h}$ old bacterial cells cultured on TSA enriched with $0.6 \%$ yeast extract were collected from the agar plates, washed once in phosphate buffered saline (PBS) ( $\mathrm{pH}$ 7.4), and suspended in the same buffer; the cell suspensions were adjusted to optical density of $0.6(450 \mathrm{~nm})$. Equal volumes $(4 \mathrm{ml})$ of the cell suspensions were centrifuged $(3,0003 \mathrm{~g}, 6 \mathrm{~min})$. The pellets were re-suspended in 500 $\mathrm{ml}$ of $1 \%$ sodium dodecyl sulfat (SDS)-Tris-HCl $(\mathrm{pH} 8)$ and shaken for $30 \mathrm{~min}$ at RT. After centrifugation, the supernatants were boiled for $5 \mathrm{~min}$ in sample buffer $(60$ $\mathrm{mM}$ Tris- $\mathrm{HCl}, \quad 1 \%$ SDS, $10 \%$ glycerol, $1 \%$ mercaptoethanol, and $0.0005 \%$ bromophenol blue) (Kotiranta et al., 1998, Kotiranta et al., 1999) and analyzed by SDS-10\% polyacrylamide gel (PAGE) electrophoresis (Sambrook et al., 2001) (Fig. 1).

Fig. 1. SDS PAGE of surface proteins in B. cereus strains Lane 1: Myosin 206 kDa-Betagalactosidase $117 \mathrm{kDa}$ - BSA $80 \mathrm{kDa}$ - Ovalbumin, $40 \mathrm{kDa}$ and Lane 2- $12:$ B. cereus strains isolated from staff hand and hospital surfaces

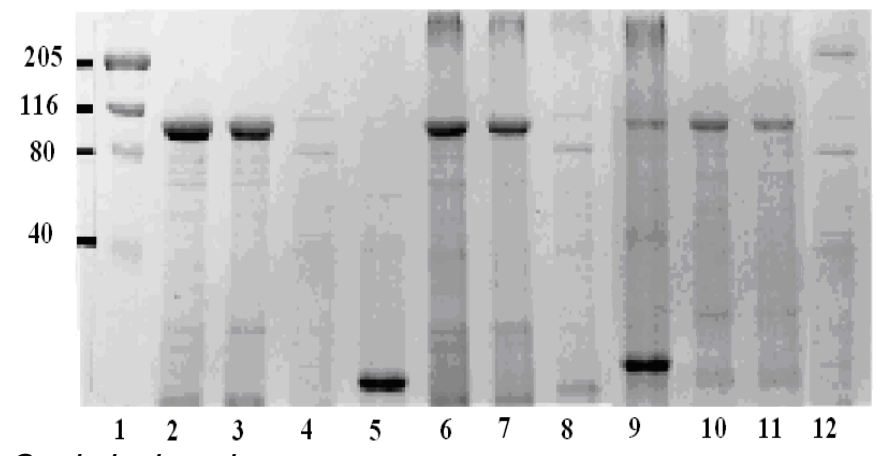

Statistical analyses

All the statistical analyses were carried out using SPSS version 14. Chi-square and fisher test was used for determination of significance of association. The $p \leq 0.05$ was considered significant.

Results and discussion

Based on the results obtained in this study, the frequency of $B$. cereus strains on hospital surfaces and staff hand was $6.7 \%$ and $16.25 \%$ respectively Based on the results of SDS-PAGE, $46.20 \%$ of the studied $B$. cereus strains have been para crystalline layer producer and $53.8 \%$ lack the ability to produce para crystalline layer (Fig. 2). Thus the $84.6 \%$ of $B$. cereus strains isolated from staff hand and $7.7 \%$ of the strains isolated from the hospitals surface have been para crystalline layer producer.

According to the results of other similar studies carried out in Iran, Bacillus species have been the most bacterial separation from the hospital environment and staff hand. Bacterial strains were isolated from hospital surface $74(24 \%)$ and from staff hands 48 (60\%) respectively (Jalalpoor et al., 2009; Jalalpoor et al., 2010; Sambrook \& Russell, 2001).

\section{Fig.2. Frequency of para crystalline layer in B. cereus strains}

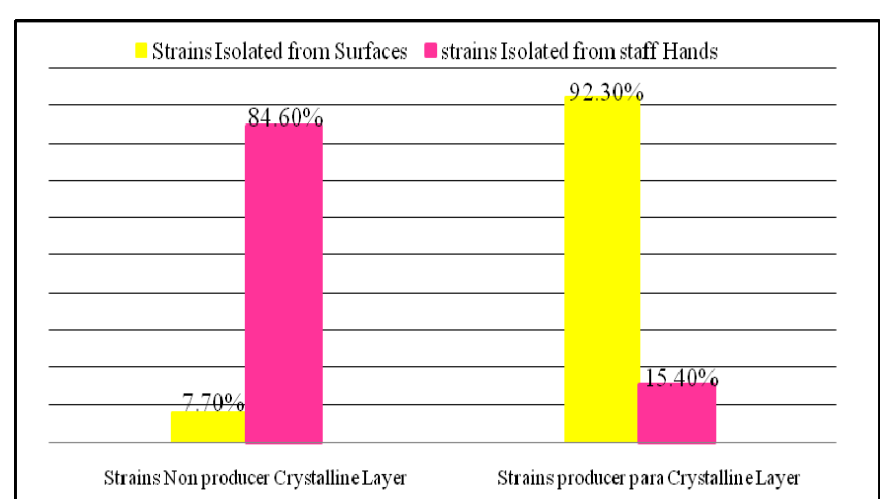

Based on the results of similar studies in other countries, the frequency of Bacillus species in staff hand was $37 \%$ and frequency of $B$. cereus strains on staff hand has been reported 15\%. In the 1998-1999 years, Kotiranta and groups studied on four strains of $B$. cereus and the strains isolated from clinical samples could produce para crystalline layer while the standard strains could not have produced para crystalline layer (Kotiranta et al., 1998; Kotiranta et al.,1999). Based on the results obtained in the present study, $11(84.60 \%)$ of $B$. cereus strains isolated from staff hand have produced para crystalline layer while only $1(7.70 \%)$ isolate from hospital surfaces was positive for para crystalline layer.

The results of this study and other similar studies, treating many of para crystalline layer in bacterial isolates from in vivo conditions, compared with bacterial isolates from in vitro conditions. Regarding this point $B$. cereus is a human pathogenic bacteria and a para crystalline layer structure is considered to be pathogenic, can be interpreted that the bacterium if considered on biological conditions, produces para crystalline layer to protect by influencing antibiotic and harmful enzymes in the human body (Jalalpoor et al., 2009; Jalalpoor et al., 2010; Sambrook \& Russell, 2001; Schaffer \& Paul, 2005).

According to results of this search and similar published study indicate spread of $B$. cereus strains resistant in hospitals, the lack of bacterial population control, leads to rapid release of resistance genes from resistance strains among sensitive bacterial population and ultimately leading to the spread of resistance nosocomial infections in hospitals and the community (Jalalpoor et al., 2010).

Acknowledgments

Author would like their subspecialty Alzahra Hospital Management, Isfahan University, Management of science research lab of Isfahan university, and all persons help us in concert to achieve this research.

\section{References}

1. Hilliard NJ, Schelonka RL and Waites KB (2003) Bacillus cereus bacteremia in a preterm neonate. $\mathrm{J}$. Clin. Microbiol. 41, 3441-3444. 
2. Jalalpoor S, Kasra Kermanshahi R, Noohi $A$ and Zarkesh Esfahani H (2007) Study of $\beta$-lactamase and S-layer layer production in some of isolated pathogen bacteria from clinical and environmental hospital samples. MSc thesis,Iran, Tehran, Islamic Azad University Science and Research Branch Tehran, Iran.

3. Jalalpoor S, Kasra Kermanshahi R, Nouhi AS, Zarkesh Esfahani H (2008) Study to Spreading Bacteria in How and Low Contact Surfaces in Hospital.9th Iranian Congress of Microbiology, Kerman, Iran. pp.208.

4. Jalalpoor S, Kasra Kermanshahi R, Nouhi AS, Zarkesh Esfahani H (2009) Survey and comparative bacterial spread pattern in staff hands and high and low contact hospital surfaces. Third Iranian Congress of Clinical Microbiology, Shiraz, Iran, pp. 184.

5. Jalalpoor S, Kasra Kermanshahi R, Nouhi AS, Zarkesh Esfahani H (2010) Survey Frequency of $\beta$ lactamase Enzyme and Antibiotic Sensitivity Pattern in Isolated Pathogen Bacteria from Low and High Hospital Contact Surfaces. Pajuhandeh J. 15, 77-82.

6. Kamp G and Kramer A (2004) Epidemiologic Background of Hand Hygiene and Evaluation of the Most Important Agents for Scrubs and Rubs. Clin. Microbiol. Rev. 17(4), 863-93.

7. Kotiranta A, Haapasalo M and Kari K (1998) Surface Structure, Hydrophobicity, Phagocytosis and Adherence to Matrix Proteins of Bacillus cereus Cells with and without the Crystalline Surface Protein Layer. Infect. Immun. 66(10), 4895-4902.

8. Kotiranta AK, Hitoshi I, Markus P, Haapasalo P and Kari L (1999) Radiation sensitivity of Bacillus cereus with and without a crystalline surface protein layer.FEMS Microbiol. Lett.179, 275-280.

9. Mesnage S, Haustant $M$ and Foue A (2001) A general strategy for identification of S-layer genes in the Bacillus cereus group :molecular characterization of such a gene in Bacillus thuringiensis subsp. galleriae NRRL 4045. J Microb.147,1343-51.

10. Messner $\mathrm{P}$, Steiner $\mathrm{K}$, Zarschler $\mathrm{K}$ and Schaffer $\mathrm{C}$ (2008) S-layer nanoglycobiology of bacteria. Carbohydr. Res. 343(12),1934-51.

11. Sambrook J and Russell DW (2001) Molecular Cloning: A Laboratory Manual, 3rd Edition. Cold Spring Harbor,NY: Cold Spring Harbor Laboratory Press.

12. Sara M (2001) Conserved anchoring mechanisms between crystalline cell surface S-layer proteins and secondary cell wall polymers in Gram-positive bacteria. Trends Microbiol. 9, 47-9.

13. Sara M and Uwe B (2000) Sleytr.S-layer Proteins. J Bacteriol. 182(4), 859-68.

14. Schaffer C and Messner P (2001) Glycobiology of surface layer proteins. Biochimie. 83, 591-99.
Vol. 5 No. S3 (Mar 2012)

ISSN: 0974- 6846

15. Schaffer $C$ and Paul M (2005) The structure of secondary cell wall polymers: how Gram-positive bacteria stick their cell walls together .Microbiol.15, 643-651.

16. Sehulster $L$ and Raymond YW (2003) Guidelines for Environmental Infection Control in Health-Care Facilities. U.S. Department of Health and Human Services Centers for Disease Control and Prevention (CDC). Atlanta GA 30333.

17. Van der Zwet WC, Parlevliet GA, Savelkoul PH, Stoof $\mathrm{J}$ and Kaiser AM (2003) Outbreak of Bacillus cereus infection in a neonatal intensive care unit traced to balloons used in manual ventilation. J. Clin. Microbiol.38, 4131-6.

18. Washington $C$, Stephen $A$, Janda $W$, Koneman $E$, Procop G, Schreckenberger P and Woods G (2006) Koneman's Color Atlas and Textbook of Diagnostic Microbiology, Sixth edition.USA: Lippincott wiiliams \& wilkins, pp: 775-9. 